\title{
Accuracy of the Chinese lunar calendar method to predict a baby's sex: a population-based study
}

\author{
Eduardo Villamor ${ }^{a}$, Louise Dekker ${ }^{b}$, Tobias Svensson $^{c}$ and Sven Cnattingius ${ }^{c}$ \\ ${ }^{a}$ Department of Environmental Health Sciences, University of Michigan School of Public Health, Ann Arbor, MI, USA, ${ }^{b}$ Department of \\ Nutrition, Harvard School of Public Health, Boston, MA, USA, and ${ }^{c}$ Clinical Epidemiology Unit, Department of Medicine, Karolinska Institute \\ and Hospital, Stockholm, Sweden
}

Correspondence:

Dr Eduardo Villamor,

Department of Environmental

Health Sciences, University of

Michigan School of Public

Health, 1420 Washington

Heights, Ann Arbor, MI

48109, USA.

E-mail: villamor@umich.edu

\section{Summary}

Villamor E, Dekker L, Svensson T, Cnattingius S. Accuracy of the Chinese lunar calendar method to predict a baby's sex: a population-based study. Paediatric and Perinatal Epidemiology 2010; 24: 398-400.

We estimated the accuracy of a non-invasive, inexpensive method (the Chinese lunar calendar, CLC) to predict the sex of a baby from around the time of conception, using 2840755 singleton births occurring in Sweden between 1973 and 2006. Maternal lunar age and month of conception were estimated, and used to predict each baby's sex, according to a published algorithm. Kappa statistics were estimated for the actual vs. the CLC-predicted sex of the baby.

Overall kappa was 0.0002 [95\% CI $-0.0009,0.0014]$. Accuracy was not modified by year of conception, maternal age, level of education, body mass index or parity. In a validation subset of 1000 births in which we used a website-customised algorithm to estimate lunar dates, kappa was -0.02 [95\% CI $-0.08,0.04]$. Simulating the misuse of the method by failing to convert Gregorian dates into lunar did not change the results. We conclude that the CLC method is no better at predicting the sex of a baby than tossing a coin and advise against painting the nursery based on this method's result.

Keywords: sex ratio, Chinese lunar calendar, month of conception.

\section{Introduction}

No methods are known to accurately predict the sex of babies from around the time of conception. Modest estimates of association have been reported between the sex ratio (males to females) at birth and 'natural' pre-conception factors, ${ }^{1-4}$ but they cannot be taken to predict the sex of any given pregnancy at the individual level. Nevertheless, some individual prediction initiatives, notably from the realms of folk belief, continue to grow in popularity. Of them, the Chinese lunar calendar (CLC) method ${ }^{5}$ appears to be among the most widely used, as suggested by Internet search statistics.

The CLC method is allegedly based on an ancient chart 'buried in a tomb near Beijing for almost 700 years', ${ }^{6}$ whose underlying rationale remains unknown. The prediction calls for two maternal variables, namely the lunar age and month at conception, which are input onto a chart from where the expected baby's sex can be read. Estimation of lunar age and month at conception from the traditional Gregorian system requires non-trivial calendrical calculations, ${ }^{7}$ and is offered by various websites. ${ }^{6,8}$

Reported accuracy rates for the CLC method range from $50 \%$ to $93 \% .^{6}$ While highly promising, these figures are not derived from thoroughly conducted epidemiological investigations. Considering the potential of this non-invasive, inexpensive method for the early prediction of a baby's sex, we undertook a formal evaluation using a population-based study.

\section{Methods}

\section{Study population}

The study population consisted of all singleton births that occurred in Sweden between 1973 and 2006, as recorded in the Swedish Medical Birth Register. From 3400212 births, we excluded records with missing 
information on the mother's date of last menstrual period ( $n=271908$ ) or the baby's sex $(n=1)$. In order to restrict the sample to mothers born within a single time zone (a potential distortion factor for the prediction, according to some reports), an additional 213196 records from non-Swedish women were excluded. Non-singleton births $(n=65250)$ and births from women outside the range of [lunar] ages at conception included in the prediction chart (18-45 years) were also excluded $(n=9102)$, for a final sample size of 2840755 births.

\section{Data analysis}

For each pregnancy, we estimated the date of conception as the date of the last menstrual period plus 14 days. Next, we obtained the maternal lunar ages and months at conception with the use of Calendrica 2.1 (Illinois Institute of Technology, IL, USA), a software package based on the algorithms by Reingold and Dershowitz. ${ }^{7}$ The predicted sex of the baby was then assigned to each woman on the basis of her lunar age and month of conception, following the CLC chart. ${ }^{6}$

The accuracy of the predicted vs. the actual baby's sex was estimated by use of the kappa statistic. ${ }^{10} \mathrm{~A}$ reasonably good kappa is expected to be $>0.45$. The estimation was made overall and within strata of maternal age, level of education, height, body mass index (BMI), pregnancy order and year of conception. We repeated the analyses restricting it to the first pregnancy only, to overcome potential within-person correlations for women with more than one pregnancy in the Register.

In order to test whether the use of formal calendrical calculations could have a distorting effect on the estimation of lunar age and month at conception, in comparison to the customised algorithms used by websites (which remained undisclosed), we selected a random sample of 1000 women and entered their data into the most popular website ${ }^{8}$ blind to the baby's actual sex, and retrieved the predicted sex. The reliability analysis was then conducted on this validation subsample.

We next tested the effects of misusing the prediction chart by entering the woman's age and month of conception in the Gregorian system, without prior transformation into lunar. This appears to be a common occurrence that websites warn against. Finally, we tested two specific assertions commonly made on the basis of the marginal probabilities of the chart, ${ }^{6}$ namely that 'the greatest odds for conceiving a boy occur when the mother conceives in July when she is $18,20,30$, and 42 ', and that 'the best odds for conceiving a girl occur when the mother conceives in April when she is 21, 22, and $29^{\prime}$, by comparing the sex ratios of births occurring at those age-and-month combinations to all others.

All analyses were conducted on a previously anonymised, de-identified dataset.

\section{Results}

Overall kappa was 0.0002 [95\% CI $-0.0009,0.0014]$. When estimated within levels of maternal characteristics (data not shown), the highest kappa was observed among women with the highest level of education, but it was low, negative and not statistically significant [-0.02; 95\% CI $-0.04,0.01]$.

Analyses of the validation subset of 1000 women revealed a rate of discrepancy in the estimation of lunar dates between the calendrical calculations and the website algorithms of $1.7 \%$; mostly due to one extra month in the calendrical estimation. Overall kappa in the validation subset was -0.02 [95\% CI $-0.08,0.04]$. In subgroup analyses of this subset, the highest value was noted in mothers under 20 years of age [-0.40; 95\% CI $-0.66,-0.14]$. Albeit statistically significant, kappa was negative in this stratum, suggesting that, if anything, the method had mild accuracy to predict the opposite sex.

Simulating the misuse of the CLC method by introducing Gregorian ages and months of conception (e.g. without prior transformation to lunar) did not substantially alter the results $[\mathrm{kappa}=0.0008 ; 95 \% \mathrm{CI}-0.0004$, 0.0019]. Finally, the sex ratios for women who conceived in July when they were 18, 20, 30 and 42 yearsold, or in April when they were 21, 22 and 29, were not significantly different than those of women who conceived at any other age or month.

\section{Discussion}

In this large, population-based study we found that the accuracy of the CLC method for the prediction of a baby's sex leaves much to be desired. Our results do not support the high rates of accuracy that have been informally reported. One potential limitation of our analysis is the use of a fixed number of days after the date of the last menstrual period to estimate the date of conception, since the length of menstrual cycles may vary. Our conception date estimation was the closest we could get at simulating what an average user of the 
CLC method would do, with her knowledge of the last menstrual period date, and an average reproductive cycle. Even if coital frequency was exactly one per cycle and the date of its occurrence was well known to the user, the exact timing of conception will still be uncertain. In addition, given that the variables used in the prediction are age and month, misclassification of conception for a few days is unlikely to cause a major bias. Bias due to the use of different algorithms in the Internet-based estimation and the formal calendrical calculations of lunar dates is also unlikely according to the results of our validation sub-study.

Lack of information on the CLC method rationale prevents additional testing. We conclude that selecting the future baby's clothes based on the results of this test would be unwise.

\section{Acknowledgements}

We are grateful to Hans J. I. Michel from Michel Information Services for assistance with advanced computer programming.

\section{References}

1 James WH. The variations of human sex ratio at birth with time of conception within the cycle, coital rate around the time of conception, duration of time taken to achieve conception, and duration of gestation: a synthesis. Journal of Theoretical Biology 2008; 255:199-204.

2 Orvos H, Kozinszky Z, Bartfai G. Natural variation in the human sex ratio. Human Reproduction 2001; 16:803.

3 Villamor E, Sparen P, Cnattingius S. Interpregnancy weight gain and the male-to-female sex ratio of the second pregnancy: a population-based cohort study. Fertility and Sterility 2008; 89:1240-1244.

4 Mathews F, Johnson PJ, Neil A. You are what your mother eats: evidence for maternal preconception diet influencing foetal sex in humans. Proceedings of the Royal Society of London Series B, Biological Sciences 2008; 275:1661-1668.

5 http://www.babygenderprediction.com/ (last accessed October 2009).

6 http://www.famouschinese.com/virtual/chinese_birth_ chart (last accessed October 2009).

7 Reingold EM, Dershowitz N. Calendrical Calculations Millenium Edition, 2nd edn. Cambridge: Cambridge University Press, 2001.

8 http://www.chinesefortunecalendar.com/predictsex.htm (last accessed October 2009).

9 Ostler S, Sun A. Fetal sex determination: the predictive vale of 3 common myths. Canadian Medical Association Journal 1999; 161: 1525-1526.

10 Cohen JA. Coefficient of agreement for nominal scales. Educational and Psychological Measurement 1960; 20:37-46. 